\title{
Resultados del uso de stent metálico autoexpandible como terapia puente en cáncer de colon izquierdo obstructivo
}

\author{
Ernesto Melkonian T. ${ }^{1}$, Eduardo Mordojovich Z. ${ }^{1}$, Antonio Rollán R. ${ }^{1}$, \\ Alex Navarro R. ${ }^{1}$, Christian Jensen B. ${ }^{1}$, Aldo Cuneo Z. ${ }^{1}$, \\ Leonardo Espíndola S. ${ }^{1}$, Daniella Espínola M. ${ }^{1}$, Militza Romagnolli R. ${ }^{2}$, \\ Sebastián Pradenas B. ${ }^{1}$, Constanza Villalón M. ${ }^{1}$ y Roque Sáenz F. ${ }^{3}$
}

\section{Results of the use of self-expanding stent as a bridge therapy in obstructive left colon cancer}

Objetive: To analyze the results of the use of stent as bridge therapy for surgery in obstructive left colon cancer in our center. Material and Method: We performed a retrospective analysis of patients with obstructive left colon cancer undergoing metallic stenting as a bridge therapy for definitive surgery between January 2008 and December 2016. Demographic data, procedural success, complications, surgical approach, pathological anatomy and follow-up were analyzed. Results: Ten patients. The procedure was technically and clinically successful in all 10 patients, with no complications in any of them. All patients went to resective surgery, achieving primary anastomosis in 9 of them. In 3 of them, a laparoscopic approach was performed. A median of lymph nodes of 41 it was obtained in the surgical specimen. With a median follow-up of 34 months, 2 presented distant recurrence, which were patients diagnosed at a more advanced stage of their disease. Discussion: The stent as bridge therapy to an elective surgery, allows to obtain a low rate of colostomies, low morbidity, offer a laparoscopic surgery and an optimal cancer surgery. Conclusion: The use of the stent as a bridge therapy in obstructive left colon cancer appears as a good alternative to solve this pathology in patients of greater surgical risk.

Key words: colorectal cancer; obstruction; stent; bridge.

\section{Resumen}

Objetivo: Analizar los resultados del uso de stent como terapia puente para cirugía en cáncer de colon izquierdo obstructivo en nuestro centro. Material y Método: Se realizó un análisis retrospectivo de pacientes con cáncer de colon izquierdo obstructivo sometidos a la instalación de stent metálicos como terapia puente para una cirugía definitiva, entre enero de 2008 y diciembre de 2016. Se analizaron datos demográficos, éxito del procedimiento, complicaciones, vía de abordaje quirúrgico, anatomía patológica y seguimiento. Resultados: Se analizaron 10 pacientes. El procedimiento fue técnica y clínicamente exitoso en los 10 pacientes, sin complicaciones. Todos fueron a cirugía resectiva, logrando anastomosis primaria en 9 pacientes. En 3 se realizó un abordaje laparoscópico. Se logró una mediana de linfonodos de 41 en la pieza quirúrgica. Con una mediana de seguimiento de 34 meses, 2 presentan recidiva a distancia siendo pacientes con etapas más avanzadas al diagnóstico de su enfermedad. Discusión: El stent como terapia de puente a una cirugía electiva, permite obtener una baja tasa de colostomías, baja morbilidad, ofrecer cirugía laparoscópica y una óptima cirugía oncológica. Conclusión: El uso del stent como terapia puente en el cáncer de colon izquierdo obstructivo aparece como una buena alternativa para resolver esta patología en pacientes de mayor riesgo quirúrgico.

Palabras clave: cáncer colorrectal; obstrucción; prótesis; puente.
'Servicio de Coloproctología, Clínica Alemana de Santiago, Facultad de Medicina Universidad del Desarrollo. Santiago, Chile.

2Residente de Cirugía, Universidad del Desarrollo, Hospital Padre Hurtado/Clínica Alemana de Santiago, Chile. 3Servicio de Gastroenterología Clínica Alemana de Santiago, Chile.

Recibido el 29 de junio de 2017 y aceptado para publicación el 7 de septiembre de 2017.

Correspondencia a: Dr. Ernesto Melkonian T. emelkonian@alemana.cl 


\section{Introducción}

El cáncer de colon izquierdo se presenta clínicamente como obstructivo en un 7 a $29 \%$ de los casos, siendo necesaria para su resolución una cirugía de emergencia, en pacientes particularmente de alto riesgo y muchas veces de edad avanzada ${ }^{1,2}$. Esta cirugía requiere en la mayoría de los casos de una colostomía y se asocia a una mayor morbilidad y mortalidad que la cirugía electiva ${ }^{2,3}$. Estos pacientes con colostomía deberán someterse a una segunda cirugía posterior para su cierre, quedando muchos de ellos con ostomía definitiva por su comorbilidad de base ${ }^{4}$.

El uso de prótesis o stent, descrito a principios de los años 90 por Dohmoto y Spinelli como paliación $^{5,6}$, fue usado como terapia puente para cirugía definitiva en 1994 por Tejero 7 . Las ventajas del stent como puente, han sido el obtener tiempo para estabilizar al paciente antes de la cirugía, preparar mecánicamente el colon y así de esta manera disminuir la necesidad de colostomía y etapificar correctamente al paciente ${ }^{8,9}$. Esta cirugía ha logrado realizarse en los últimos años, incluso por vía laparoscópica ${ }^{10}$.

Pese a las ventajas descritas, se han reportado diferentes cifras de éxito en su uso y existe aún debate sobre su resultado oncológico y, por tanto, su verdadero rol.

Su utilización ha sido descrita en un reporte nacional, que incluye pacientes en quienes se utilizó stent principalmente como terapia paliativa.

\section{Objetivo}

Analizar los resultados técnicos y quirúrgicos del uso de stent, instalado como terapia puente en la cirugía en cáncer de colon izquierdo obstructivo, sus complicaciones y resultado oncológico en nuestro centro.

\section{Material y Método}

Se realizó un análisis retrospectivo de los procedimientos realizados en Clínica Alemana de Santiago entre el 1 de enero de 2008 al 31 de diciembre de 2016, de pacientes consecutivos que presentaron obstrucción intestinal baja de origen neoplásico en colon izquierdo, que no presentaran signos de perforación, y que fueron sometidos a la instalación de stent autoexpandibles, para solucionar la obstrucción con la intención de utilizarlo como terapia puente a la cirugía definitiva.

Todos los pacientes aceptaron ser sometidos al procedimiento, mediante un documento de con- sentimiento informado. El Comité de Ética de la institución, autorizó el uso de los datos clínicos de los registros electrónicos de los pacientes, en forma anónima.

Todos los pacientes fueron estudiados con tomografía computada al momento del diagnóstico. Se definió cáncer de colon izquierdo, a aquellos ubicados desde el colon transverso izquierdo hasta la unión recto-sigmoidea. Se excluyeron del análisis, los pacientes a los cuales se realizó instalación de stent en contexto paliativo y un paciente de edad avanzada que rechazó la cirugía posterior a instalación de la prótesis.

Se utilizó stents autoexpandibles no cubiertos Hanarostent de M. I. Tech y WallFlex de Boston Scientific de tamaños variables (desde $90 \times 25 \mathrm{~mm}$ hasta $110 \times 24 \mathrm{~mm}$ ) según características del tumor, instalados por un grupo acotado de endoscopistas experimentados en el uso de endoprótesis de nuestra institución (Figura 1). Los procedimientos fueron realizados bajo sedación anestésica administrada por anestesiólogo. Se define descompresión técnicamente exitosa, como el paso de la guía y la instalación del stent correctamente a la fluoroscopia (Figura 2). El éxito clínico está dado por la descompresión (paso de gas y materia fecal) de la obstrucción de colon dentro de las primeras $72 \mathrm{~h}$ posinstalación del stent ${ }^{12}$.

A todos los pacientes se les realizó preparación mecánica del colon con Fleet oral ${ }^{(\mathrm{R})} 2$ frascos posdescompresión endoscópica, previo a la cirugía.

Todas las piezas quirúrgicas fueron enviadas posteriormente a estudio histológico (Figura 3).

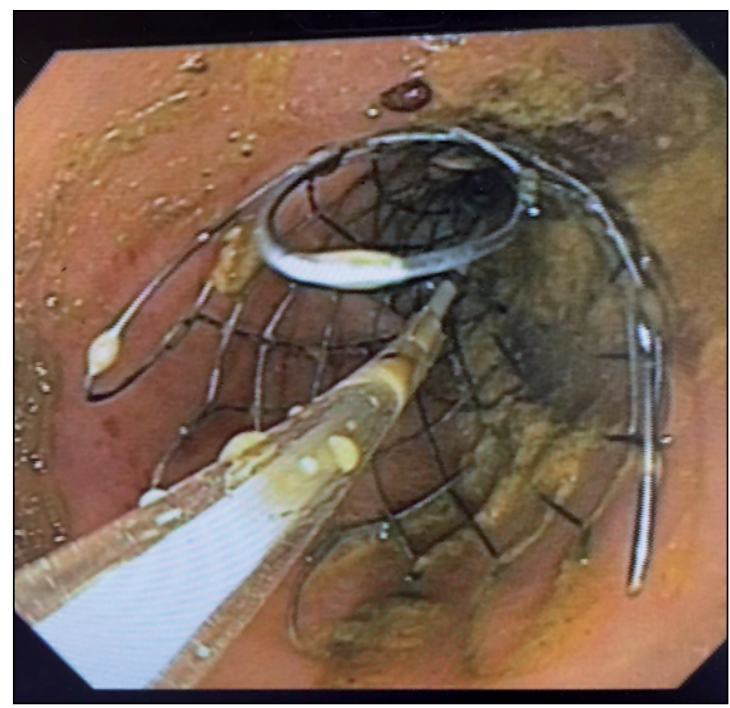

Figura 1. Stent autoexpandible in situ. 
Se analizaron datos demográficos, éxito del procedimiento endoscópico y posterior manejo, vía de abordaje quirúrgico y seguimiento de los pacientes descritos, desde la perspectiva clínica y oncológica.

\section{Resultados}

Entre enero de 2008 y diciembre de 2016 se reclutaron 10 pacientes tratados con stent de colon como terapia puente para cirugía electiva. Seis de sexo femenino y 4 masculino, con una mediana de edad de 65 años (85-37 años). La localización más frecuente del tumor fue en colon sigmoides en 7 casos, seguido 2 casos de colon izquierdo y 1 en colon transverso distal.

La descompresión colónica con stent fue técnica y clínicamente exitosa en el $100 \%$ de los pacientes. No se reportaron complicaciones relacionadas al stent en ninguno de los pacientes. No hubo perforaciones o migración del dispositivo en el período preoperatorio.

De los 10 pacientes, 2 presentaban etapa IV al momento del diagnóstico (metástasis hepáticas en ambos casos). Desde la instalación exitosa del stent hasta la cirugía resectiva de colon transcurrieron una mediana de 7 días, con un máximo de 74 días y un mínimo de 2 días. Los pacientes que tuvieron prótesis instalada por más días (74 y 68 días), debieron ser sometidos al tratamiento de metástasis hepáticas con quimioterapia sistémica y cirugía hepática previo a la cirugía de colon.

De los 10 pacientes, en 3 se logró abordaje laparoscópico sin incidentes. En 9 pacientes se realizó una anastomosis primaria y sólo un paciente requirió ser ostomizado (operación de Hartmann), por demostrar en el intraoperatorio que la descompresión de colon fue insuficiente.

No hubo dehiscencia anastomótica ni mortalidad en la serie. Dos pacientes presentaron complicaciones: un seroma de herida operatoria y una colección serosa pélvica drenada por vía percutánea.

Con respecto a la anatomía patológica y número de linfonodos resecados, se obtuvo una mediana de 41 linfonodos resecados, (máximo de 78 y mínimo 31 linfonodos). Al etapificar a los pacientes, 1 fue catalogado como etapa I, 4 como etapa II, 3 pacientes en etapa III y 2 pacientes como etapa IV.

Los pacientes incluidos en esta serie de casos fueron seguidos en el 100\%, con una mediana de 34 meses. De los 10 pacientes, 4 fallecieron durante el seguimiento: 2 producto del cáncer de colon, uno de ellos diagnosticado inicialmente con metástasis

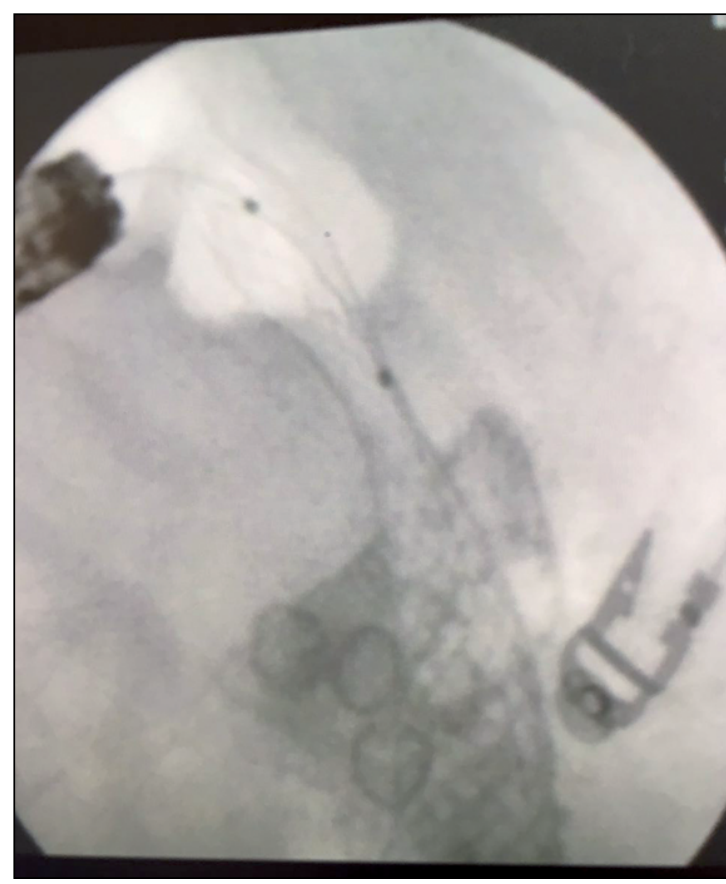

Figura 2. Control de stent por fluoroscopia.

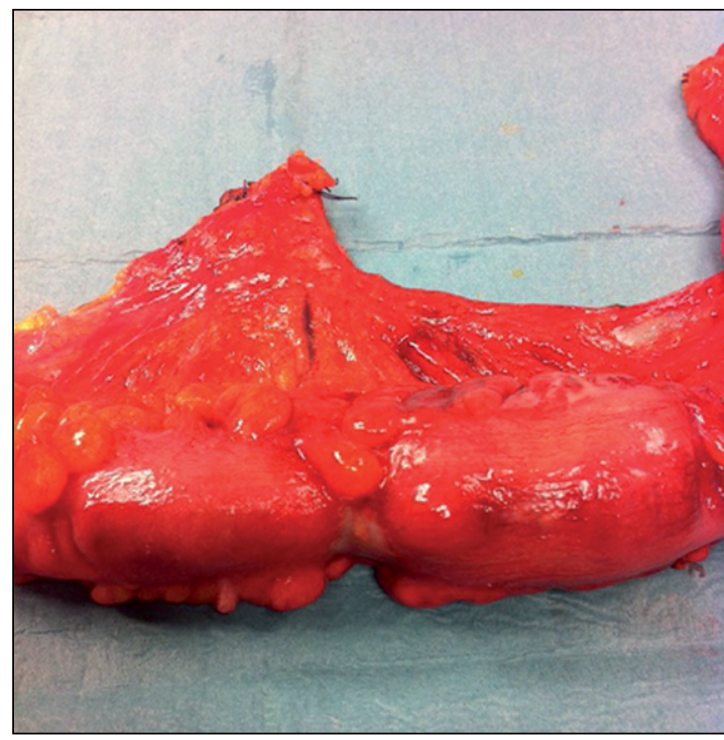

Figura 3. Pieza anatómica.

hepáticas sincrónicas y el otro paciente que presentaba un tumor con invasión de intestino delgado y que rechazó quimioterapia posoperatoria. Otros 2 pacientes fallecieron por causas no relacionadas con el cáncer de colon. Los 6 pacientes restantes, se mantienen en seguimiento periódico sin recidiva. Tabla 1). 
Tabla 1. Pacientes operados con stent de colon como puente a cirugía

\begin{tabular}{|ccccccccc|}
\hline $\begin{array}{c}\text { Paciente } \\
\mathbf{N}^{\mathbf{0}}\end{array}$ & Género* & Edad & Tumor & Estadio & Abordaje & A/O*** & $\begin{array}{c}\text { Linfonodos } \\
\text { resecados }\end{array}$ & Seguimiento \\
\hline 1 & F & 74 & Descendente & IIB & Abierta & A & 33 & Sin recidiva \\
\hline 2 & M & 69 & Rectosigmoides & IIIB & Lap** & A & 39 & Sin recidiva \\
\hline 3 & F & 37 & Sigmoides & IIA & Abierta & A & 60 & Sin recidiva \\
\hline 4 & F & 74 & Sigmoides & IIC & Abierta & O & 44 & Fallece por cáncer de colon \\
\hline 5 & F & 85 & Sigmoides & IIA & Abierta & A & 34 & Fallece por otra causa \\
\hline 6 & F & 78 & Transverso distal & IIIB & Abierta & A & 76 & Fallece por otra causa \\
\hline 7 & F & 47 & Sigmoides & IVA & Lap** & A & 57 & Fallece por cáncer de colon \\
8 & M & 58 & Descendente & IIIB & Lap** & A & 31 & Sin recidiva \\
\hline 9 & M & 60 & Sigmoides & IVA & Abierta & A & 39 & Sin recidiva \\
10 & M & 55 & Sigmoides & I & Abierta & A & 78 & Sin recidiva \\
\hline
\end{tabular}

*M: Masculino; F: Femenino. **Lap: Laparoscópico. ***A/O: Anastomosis/Ostomía.

\section{Discusión}

La cirugía de emergencia para resolver el cáncer de colon izquierdo obstructivo se asocia a una alta morbilidad (32-64\%) y mortalidad (15-34\%) $)^{2,3,13}$.

El uso del stent nació inicialmente para el tratamiento endoscópico de patologías malignas esofágicas y estenosis biliares. Con los años se consideró de utilidad en el manejo del cáncer de recto y sigmoides obstructivo. Fue Dohmoto el primero en publicar su uso paliativo el año $1991^{5,8}$. Múltiples publicaciones han confirmado su utilidad en el contexto paliativo. Desde 1994 a la fecha, también se han publicado experiencias acerca de su utilidad como terapia puente, para una cirugía definitiva, sin embargo, está aún en duda su indicación precisa debido a la posibilidad de ensombrecer el pronóstico oncológico $^{14}$.

Al analizar esta serie de casos, se lograron objetivar varios beneficios, previamente descritos. En nuestra serie, a todos los pacientes se les logró preparar el colon una vez instalado el dispositivo, lo que permitió realizar una anastomosis primaria en 9 de los 10 pacientes. Es decir, se logró evitar la colostomía en la mayoría de los pacientes, evitando la morbilidad y disminución de la calidad de vida asociada, como también una segunda cirugía para el cierre de la ostomía. Este ítem también lo confirma van den Berg y colaboradores al comprobar una menor necesidad de ostomías en pacientes con stent, en cáncer de colon izquierdo obstructivo, comparados con pacientes con cirugía primaria ${ }^{15}$.

Es importante lograr una tasa de descompresión adecuada, que debe ser mayor del $92 \%$ para justificar la utilidad de este procedimiento ${ }^{12,16}$.

Otro beneficio observado de su uso, es la posibilidad de una mejor cosecha de linfonodos en la pieza quirúrgica $\mathrm{y}$, por lo tanto, una cirugía oncológica adecuada, lo que probablemente se deba al hecho de trabajar con un colon menos distendido, facilitándose así la disección y en parte por realizarse la cirugía electiva por un equipo especializado ${ }^{17}$.

En esta serie de casos se logró resecar más de 12 linfonodos en el $100 \%$ de nuestros pacientes con una mediana de 41. Existen reportes que han demostrado que transformando la cirugía en electiva de esta manera, se logra recolectar un mayor número de linfonodos, comparado a la cirugía de urgencia ${ }^{14,18}$. Tung y colaboradores presentan una mediana de 23 linfonodos en cirugía con stent versus 11 linfonodos en cirugía de urgencia, con un $\mathrm{p}$ significativo ${ }^{18}$.

Una tercera ventaja del uso de stent es el poder realizar una cirugía mediante abordaje laparoscópico. Gorissen y colaboradores demuestran que existe una diferencia significativa a favor del uso de la laparoscopia en el grupo con stent comparado con el grupo de cirugía de urgencia ${ }^{1}$. En nuestro grupo de pacientes se logró sin inconvenientes, el abordaje laparoscópico en 3 pacientes. La utilización de esta técnica en nuestra serie dependió exclusivamente de la preferencia del cirujano tratante.

Pensamos que tanto transformar la cirugía de urgencias en electiva, como el abordaje laparoscópico en un tercio de los casos, pueden haber contribuido a la baja morbilidad de nuestra serie $(20 \%)$, en 
comparación a las cifras esperadas de la cirugía de emergencia.

El aspecto más controversial acerca del uso del stent como terapia puente, en el cáncer de colon izquierdo obstructivo, es el beneficio o perjuicio oncológico. Múltiples trabajos han intentado dilucidar la influencia del stent sobre el pronóstico oncológico, con resultados contradictorios, aunque la mayoría no encuentra diferencias significativas. Li y colaboradores publican sus resultados comparando un grupo de cirugía electiva luego del uso de stent, versus un grupo que fue directamente a cirugía de urgencia $^{19}$. Ellos demuestran beneficio oncológico a favor del primer grupo, con una sobrevida de 53 meses versus 41 meses en el grupo de cirugía de urgencia, con un $\mathrm{p}=0,034$.

Tung y colaboradores en un trabajo prospectivo, randomizado, compararon pacientes estadio II y III, sometidos a resección curativa en cáncer de colon obstructivo, no encontrando diferencias significativas en sobrevida a 5 años al comparar los grupos (42,8\% en cirugía de urgencia versus $57,1 \%$ en cirugía con stent, con un $\mathrm{p}=0,347)^{18}$. Resultado similar muestra Zhang en su metaanálisis publicado el año 2012, así como un reciente trabajo randomizado múlticéntrico ${ }^{8,17}$.

Algunas publicaciones muestran un resultado oncológico adverso con el uso de stent de colon, principalmente Sabbagh y colaboradores, quienes observan que, con su uso, tienen peor sobrevida a 5 años que la cirugía de urgencia (25\% versus $62 \%$ a 5 años con un p de 0,0003) ${ }^{14}$. Este resultado, sin embargo, presenta sesgos, ya que el porcentaje de pacientes en etapa IV del primer grupo, es de $37,5 \%$ versus $4 \%$ del grupo de cirugía de urgencia $(p=0,0154)$. En este mismo trabajo se compara los grupos excluyendo los pacientes etapa IV y pacientes con perforación al diagnóstico, persistiendo la diferencia significativa a favor de la cirugía de urgencia. Sin embargo, como trabajo retrospectivo, tiene limitaciones en sus resultados. Gorissen y colaboradores, demuestran mayor recurrencia local en pacientes menores de 75 años en quienes se instaló un stent y fueron operados en forma electiva (32\% recurrencia local en grupo stent versus $8 \%$ en grupo de cirugía urgencia con un $\mathrm{p}=$ 0,038). Sin embargo, no logró demostrar diferencia significativa de sobrevida entre ambos grupos ${ }^{1}$.

También se ha evaluado el resultado oncológico, en pacientes con perforación de colon luego de la instalación de un stent. Sloothaak, compara la sobrevida libre de enfermedad en pacientes con perforación de colon con y sin complicaciones luego de la instalación ${ }^{20}$. Concluye que existe peor resultado oncológico en los pacientes perforados que en los no perforados, con una sobrevida libre de enfermedad de $0 \%$ versus $45 \%$ a 4 años respectivamente $(p=0,007)$, pero sin impacto en la sobrevida global.

Es importante señalar que la tasa de perforación de Sloothaak y colaboradores es del $23 \%$. Se recomienda una tasa de perforación máxima de $8 \%$, para no empeorar el resultado oncológico9. Es interesante acotar que estudios multicéntricos, como el publicado por van Hooft ${ }^{22}$, tienen menor tasa de éxito y mayor tasa de perforación en la instalación de la prótesis, habiendo un grupo diverso de endoscopistas. En nuestra serie, tratados por un grupo acotado de endoscopistas expertos en endoprótesis, no se reportaron casos de perforación. Pensamos que nuestros buenos resultados oncológicos están dados por el buen éxito del procedimiento endoscópico.

Es recomendable realizar la cirugía lo más precoz posible, una vez descomprimido el colon, para evitar complicaciones como perforación o migración, lo que en nuestra serie ocurrió con una mediana de 7 días posprocedimiento, similar a cifras reportadas en otras series ${ }^{16}$.

Algunos autores han recomendado reservar esta técnica para pacientes de más de 70 años o ASA III o más, ya que así lograrían el mejor beneficio al bajar la morbimortalidad y evitar el riesgo de una eventual recidiva local ${ }^{21,22}$.

Nuestra serie, aunque pequeña, tiene una mediana de edad alta y refleja los pacientes consecutivos de nuestro centro manejados con endoprótesis y que no presentaban obstrucción de colon derecho ni tumores obstructivos perforados ni manejo paliativo. A nuestro parecer, en este grupo de pacientes, esta es la técnica de elección para esta patología dado nuestros buenos resultados técnicos.

De nuestros pacientes así tratados que fueron a cirugía resectiva, sólo 2 han fallecido producto del cáncer de colon con una mediana de seguimiento de 34 meses siendo pacientes con cáncer en etapas avanzadas, por lo que no parece haber un resultado oncológico desfavorable en nuestra serie.

Aún no hay series nacionales publicadas sobre stent como terapia puente para comparar nuestros resultados, sin embargo, creemos poder concluir que su uso como terapia puente a la cirugía para el cáncer de colon izquierdo obstructivo, aparece como una buena alternativa para resolver esta patología especialmente en pacientes de edad avanzada o con patologías asociadas, presentando baja morbilidad, una óptima cirugía oncológica y una baja tasa de requerimiento de colostomía. Creemos que es importante contar con un alto porcentaje de éxito en el procedimiento, para no perjudicar el resultado clínico y oncológico. 


\section{Responsabilidades éticas}

Protección de personas y animales. Los autores declaran que para esta investigación no se han realizado experimentos en seres humanos ni en animales.

Confidencialidad de los datos. Los autores declaran que han seguido los protocolos de su centro de trabajo sobre la publicación de datos de pacientes.

Derecho a la privacidad y consentimiento informado. Los autores han obtenido el consentimiento informado de los pacientes y/o sujetos referidos en el artículo. Este documento obra en poder del autor de correspondencia.

\section{Financiación}

Los autores declaran no haber recibido ninguna financiación para la realización de este trabajo.

\section{Conflicto de intereses}

Los autores declaran no tener ningún conflicto de intereses.

\section{Bibliografía}

1. Gorissen KJ, Tuynman JB, Fryer E, Wang L, Uberoi R, Jones OM et al. Local recurrence after stenting for obstructing left-sided colonic cancer. Br J Surg. 2013;100:1805-9.

2. Smothers L, Hyan L, Fleming J, Tunage R, Simmang, Anthony T. Emergency surgery for colon carcinoma. Dis Colon Rectum 2003;46:24-30.

3. Amri R, Bordeianou L, Sylla P, Berger D. Colon cancer surgery following emergency presentation: effects on admission and stage-adjusted outcomes. Am J Surg. 2015;209: 246-53.

4. Horesh N, Dux JY, Nadler M, Lang A, Zmora O, Shacham-Shmueli E, et al. Stenting in malignant colonic obstructionis it a real therapeutic option? Int J Colorectal Dis. 2016;31: 131-5.

5. Dohmoto M, Rupp KD, Hohlbach G. Neue endoskopische Verfahren zur Palliation beim Rektumcarcinom. In Büntett, Jungingerth, eds. Jahrbuch der Chirurgie. Münster, Biermann Verlag, 1992, 109-15.

6. Spinelli P, Dal Fante M, Mancini A. Selfexpanding mesh stent for endoscopic palliation of rectal obstructing tumors: a preliminary report. Surg Endoscopy 1992; 6:72-4.

7. Tejero E, Mainar A, Fernández L, Tobío $\mathrm{R}$, De Gregorio MA. New procedure for the treatment of colorectal neoplastic obstructions. Dis Colon Rectum 1994;37:1158- 9.

8. Zhang Y, Shi J, Shi B, Song CY, Xie WF, Chen YX. Self-expanding metallic stent as a bridge to surgery versus emergency surgery for obstructive colorectal cancer: a metaanalysis. Surg Endosc. 2012;26:110-9.

9. Suárez J, Jiménez-Pérez J. Long-term outcomes after stenting as a"bridge to surgery" for the management of acute obstruction secondary to colorectal cancer. World J Gastrointest Oncol. 2016; 8:10512.

10. Gianotti L, Tamini N, Nespoli L, Rota M, Bolzonaro E, Frego R, et al. A prospective evaluation of short-term and long-term results from colonic stenting for palliation or as a bridge to elective operation versus immediate surgery for large-bowel obstruction. Surg Endosc. 2013;27:83242.

11. Fava M, Contreras O, Loyola S, Lopez F. Neoplasia colorrectal. Tratamiento de la obstrucción con endoprótesis metálicas autoexpandibles. Rev Med Chile 1999;127(:1207-12.

12. Watt AM, Faragher IG, Griffin TT, Rieger NA, Maddern GJ. Self-expanding metallic stents for relieving malignant colorectal obstruction: a systematic review. Ann Surg. 2007;246:24-30

13. De Salvo GL, Gava C, Pucciarelli S, Lise M. Curative surgery for obstruction from primary left colorectal carcinoma: primary or staged resection? Cochrane Database Syst Rev. 2004;2.

14. Sabbagh C, Browet F, Diouf M, Cosse C, Brehant $\mathrm{O}$, Bartoli $\mathrm{E}$, et al. Is stenting as "a Bridge to Surgery" an oncologically safe strategy for the management of acute, left-sided, malignant, colonic obstruction? A comparative study with a propensity score analysis. Ann Surg. 2013;258:10715.

15. van den Berg MW, Sloothaak DA, Dijkgraaf MG, van der Zaag ES, Bemelman WA, Tanis PJ et al. Bridge-tosurgery stent placement versus emergency surgery for acute malignant colonic obstruction. Br J Surg. 2014;101:867-73.

16. Kwak MS, Kim WS, Lee JM, Yang DH,
Yoon YS, Yu CS, et al. Does stenting as a bridge to surgery in left- sided colorectal cancer obstruction really worsen oncologic outcomes? Dis Colon Rectum 2016;59:725-32

17. Arezzo A, Balague C, Targarona E, Borgh F, Giraudo G, Ghezzo L, et al. Colonic stenting as a bridge to surgery versus emergency surgery for malignant colonic obstruction: results of a multicentre randomised controlled trial (ESCO trial). Surg Endosc. 2017;31:3297-305.

18. Tung KL, Cheung HY, Ng LW, Ching CC, Li MK. Endo-laparoscopic approach versus conventional open surgery in the treatment of obstructing left-sided colon cancer: Long-term follow up of a randomized trial. Asian J Endosc Surg. 2013;6:78-81.

19. Li ZX, Wu XH, Wu HY, Chang WJ, Chang XJ, Yi T, et al. Self-expandable metallic stent as a bridge to elective surgery versus emergency surgery for acute malignant colorectal obstruction. Int J Colorectal Dis. 2016;31:561-70.

20. Sloothaak DA, van den Berg MW, Dijkgraaf MG, Fockens P, Tanis PJ, van Hooft JE, et al. Oncological outcome of malignant colonic obstruction in the Dutch stent-In 2 trial. Br J Surg. 2014;101:1751-7.

21. Centikaya E, Dogrul AB, Timakiz MB. Role of expandable stents in management of colorectal cancers. World J Gatrointest Oncol. 2016;8:113-20.

22. van Hooft JE, van Halsema EE, Vanbiervliet G, Beets-Tan RG, DeWitt JM, Donnellan F, et al. Self-expandable metal stents for obstructing colonic and extracolonic cancer: European Society of Gastrointestinal Endoscopy (ESGE) Clinical Guideline. Gastrointest Endosc 2014;80:747-61. 